\title{
Method to Filter ECGs and Evaluate Clinical Parameter Distortion using Realistic ECG Model Parameter Fitting
}

\author{
GD Clifford $^{1}$, PE McSharry ${ }^{2,3}$ \\ ${ }^{1}$ Harvard-MIT Division of Health Sciences \& Technology, 45 Carleton St., Cambridge, MA, USA \\ ${ }^{2}$ Department of Engineering Science, \& Mathematical Institute, University of Oxford, Oxford, UK \\ ${ }^{3}$ Centre for the Analysis of Time Series, London School of Economics, London, UK
}

\begin{abstract}
By fitting a previously published nonlinear model for generating realistic ECG to waveforms collected from a healthy human subject, and using a nonlinear leastsquares optimization procedure, the authors demonstrate that significant points ( $P, Q, R, S$, and $T)$ on the ECG can be determined to an arbitrary accuracy. The model-fitting routine runs in real-time on a $3 \mathrm{GHz} P$. Coloured $\left(1 / f^{\beta}\right)$ noise is then added to the ECG in order to evaluate the fitting accuracy under a variety of recording conditions. A method for determining noise levels (and colour) in real ECGs using the residual of a singular valued decomposition is described. Furthermore, a method for evaluating the filter is described which allows an application-specific evaluation of the filter in terms of the distortion in the QRS width and amplitude, the ST-level, the QT interval, the PR-interval, and the fiducial point location. Using these methods, the model-based filter is shown to introduce insignificant clinical distortion in the QT interval and $Q R S$ width down to an $S N R \geq 0 d B$ for $\beta<2$. The fiducial point location is shown to be insignificantly distorted $(<1 \mathrm{~ms})$ for an $S N R \geq 2 d B$, and the ST-level is stable down to $S N R>12 d B$. PR interval is more sensitive to noise due to the low amplitude nature of the $P$-wave. In general, the filter performance is degraded by increasing $\beta$.
\end{abstract}

\section{Introduction}

Existing techniques for filtering and segmenting ECGs are limited by the lack of an explicit patient-specific model to help isolate the required signal from contaminants. Only a vague knowledge of the frequency band of interest and almost no information concerning the morphology of an ECG are generally used. Previously proposed adaptive filters [1, 2], require another reference signal, or some ad-hoc generic model of the signal as an input.

In this paper a gradient descent method for deriving the parameters of a previously described realistic ECG model
[3] on a beat-by-beat basis for an arbitrary ECG is detailed. This represents an alternative filtering paradigm wherein a patient specific model of the ECG is used to form a noisefree representation of a subject's ECG. No prior knowledge of the morphology and only one channel of the ECG is required. Furthermore, the locations of each peak, plus their onsets and offsets can be completely known and beat segmentation is therefore possible.

A method for calculating the noise power and colour in an ECG based on Singular Value Decomposition (SVD) is then presented, followed by a assessment of the fitting performance as a function of noise power and colour for standard clinical parameters (surrogates for the PR-interval, QT-interval, QRS-width, ST-level and RS-amplitude). Using this method, a researcher can then a priori predict the performance of the model-fit for a given ECG segment and for a particular clinical application, hence facilitating the automatic rejection of beats where the clinical distortion may be significant. Illustrations of applications of the model-fitting procedure, including ECG segmentation, filtering, compression, classification are then discussed. Finally a discussion of possible improvements is presented.

\section{Methods}

\subsection{The signal model}

The model presented here is essentially a non-dynamic version of our original model [3, 4] with the inclusion of one extra parameter per asymmetric wave (only the Twave in this paper). Each symmetrical feature of the ECG $(P, Q, R, \& S)$ is described by a Gaussian with three parameters; the amplitude $a_{i}$, width $b_{i}$ and phase $\theta_{i}=2 \pi / t_{i}$ (or relative position with respect to the R-peak). Since the $T$-wave is often asymmetric, it is described by the sum of two Gaussians (and hence 6 parameters) and is denoted by a superscripted - or + , to indicate that they are located at values of $\theta$ (or $t$ ) slightly either side of the peak of the $T$ wave (the original $\theta_{T}$ for a for the symmetric model). The 
vertical displacement of the ECG, $z$, from the isoelectric line (at an assumed value of $z=0$ ) is then described by an ordinary differential equation,

$$
\dot{z}\left(a_{i}, b_{i}, \theta_{i}\right)=\sum_{i \in\left\{P, Q, R, S, T^{-}, T^{+}\right\}} a_{i} \Delta \theta_{i} e^{\left(\frac{-\Delta \theta_{i}^{2}}{2 b_{i}^{2}}\right)}
$$

where $\Delta \theta_{i}=\left(\theta-\theta_{i}\right)$, the relative phase. Numerical integration of this equation using appropriate set of $a_{i}, b_{i}$ and $\theta_{i}$ leads to the familiar ECG waveform.

\subsection{Fitting parameters to the model}

One efficient method of fitting the ECG model described above to an observation $s(t)$, is to minimize the squared error between the $s$ and $z$. That is, we wish to find error

$$
\varepsilon_{r}=\min _{a_{i}, b_{i}, \theta_{i}}\|s(t)-z(t)\|_{2}^{2}
$$

over all six $i$. Fortunately, we can analytically integrate (1) to give $z\left(a_{i}, b_{i}, t_{i}\right)=\sum_{i} 2 a_{i} \Delta \theta_{i} \exp \left(-\Delta \theta_{i}^{2} / 2 b_{i}^{2}\right)$. Equation (2) can then be solved using an eighteen-dimensional nonlinear gradient descent on the parameter space [5]. To minimize the search space for fitting the parameters $\left(a_{i}, b_{i}\right.$, and $\theta_{i}$ ), a simple peak-detection and time-aligned averaging technique is performed to form an average beat morphology using at least the first 60 beats centered on their R-peaks. (The template window length is unimportant, as long as it contains all the $P Q R S T$ features and does not extend into the next beat). This method, including outlier rejection is detailed in [6]. $T^{-}$and $T^{+}$are initialized $\pm 40 \mathrm{~ms}$ either side of $\theta_{T}$. By measuring the heights of each peak (or trough) an estimate of the $a_{i}$ can also be made. Each $b_{i}$ is initialized with a value $10+5 \mu$, where $\mu$ is a uniform distribution on the interval $[0, \ldots, 1]$. Each of the $a_{i}$ and $\theta_{i}$, were initialized with random perturbations of $\mu$ and $20 \mu$ respectively. Figure 1 illustrates an example of a template ECG, the model-fit, and the residual error, $\varepsilon_{r}$.

Note that it is important that salient features that one wishes to fit (the P-wave and QRS segment in the case of the ECG) are sampled at a high enough frequency to allow them to contribute sufficiently to the optimization. In empirical tests it was found that when $F_{s}<450 \mathrm{~Hz}$, upsampling is required (using an appropriate anti-aliasing filter). With $F_{s}<450 \mathrm{~Hz}$ there are often fewer than 30 sample points in the QRS complex and this can lead to some extremely bizarre fits that still fulfill the optimization criteria.

Figure 2 illustrates a sequential fit of the model (upper plot) to a series of noisy ECG beats (lower plot) and the residual error of the fit (central plot, pink noise).

\subsection{Clinical metrics, colour \& SNR}

ECG data was recorded at $256 \mathrm{~Hz}, 12$ bit from the author and the surrogates for the QT-interval, PR-interval, QRS

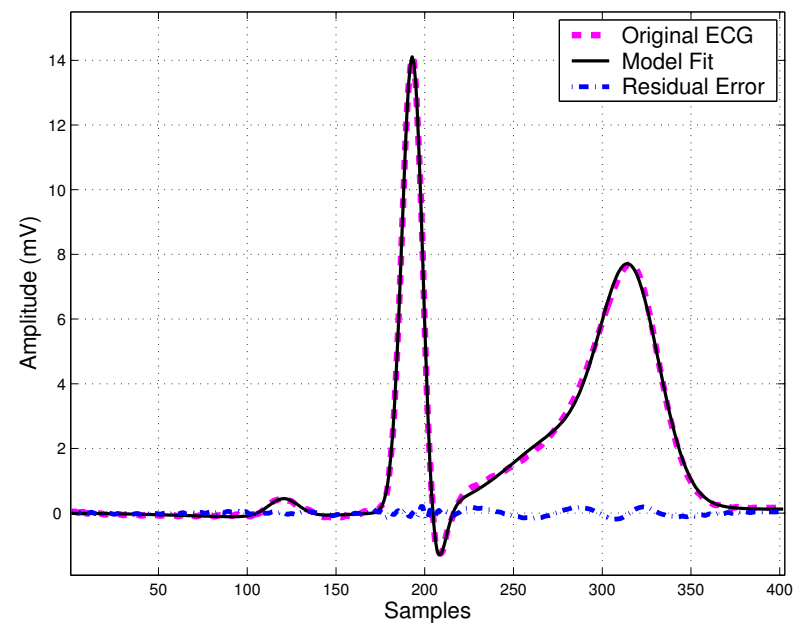

Figure 1. Original ECG, model fit, and residual error.

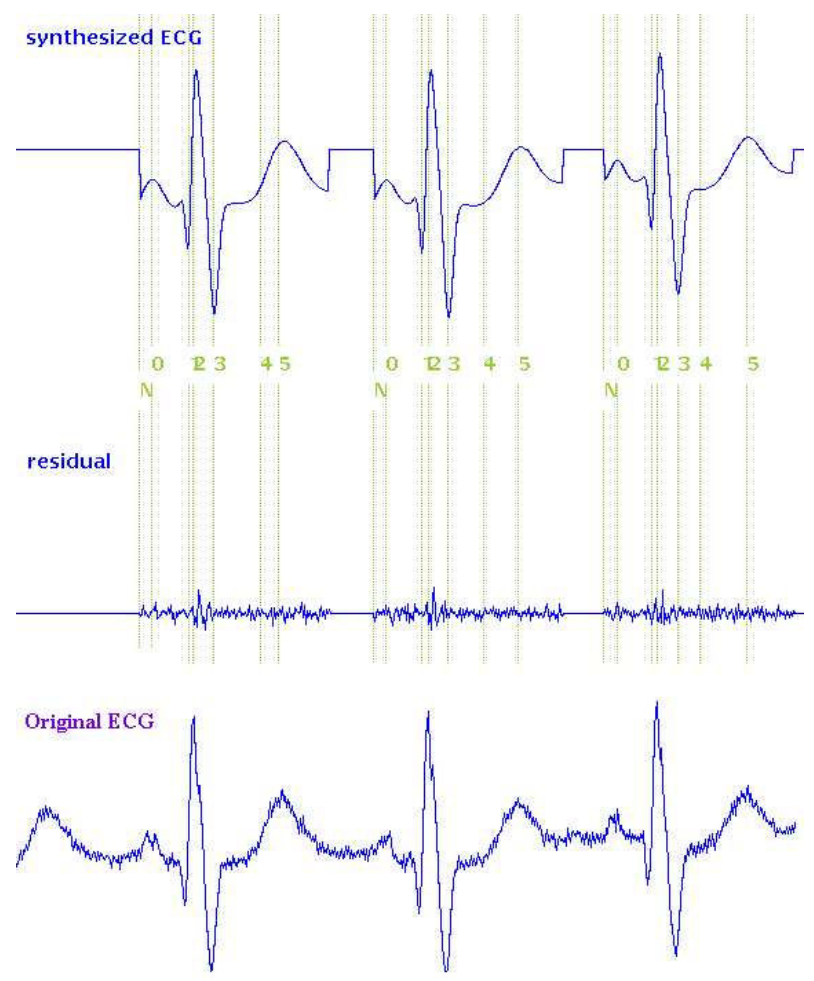

Figure 2. Segmentation of the ECG using the model-fit procedure ( $3 \mathrm{~s}$ window). $N$ indicates the onset of the segmentation region and the labels $\{0, \ldots, 5\}$ indicate the $\theta_{i}$. 
width, RS amplitude and ST-level were derived from an averaged (virtually noise free) template made form averaging the first 60 sinus beats. For simplicity, the surrogate clinical values are defined such that they can be extracted by taking differences in pairs of $\theta_{i}$ (or ECG amplitudes at the locations of the $\theta_{i}$ ) which resulted in negligible error in the clinical parameters. $1 / f^{\beta}$ noise (white $\beta=0$, pink $\beta=1$ and brown $\beta=2$ ) was then added at incrementally higher signal-to-noise ratios (SNRs). Each clinical parameter was measured after model-fitting at each SNR.

The ST level was measured at $80 \mathrm{~ms}$ after the $\theta_{S}$. Clinically significant distortion in the ECG is defined by the Sheffield criteria [7] to be $\geq 0.1 \mathrm{mV}$ for the ST-level (or about $5 \%$ to $10 \%$ of the QRS amplitude for a sinus beat on a V5 lead). For fiducial (R-) peak location $\left(\theta_{R}\right)$, HRV studies have shown that sampling frequencies below $0.5-1 \mathrm{kHz}$ may distort HRV estimates [8] and therefore a change above $1 \mathrm{~ms}$ is considered significant. The QT interval is the time from onset of QRS to end of T wave (divided by the square root of the heart rate). Our surrogate QT interval $2 \pi /\left(\theta_{T}-\theta_{Q}\right)$. A normal male QT-interval is about $0.39 \pm 0.04 \mathrm{~s}$ (with slightly larger values for women). Values over $0.5 \mathrm{~s}$ are cause for concern, therefore we consider $0.05 \mathrm{~s}$ as a clinically significant distortion.

The PR interval extends from the start of the $\mathrm{P}$ wave to the start of the QRS complex (i.e., to the start of the Q wave). A normal value is $0.12 \mathrm{~s}$ to $0.20 \mathrm{~s}$. The surrogate PR interval used here $\left(2 \pi /\left(\theta_{R}-\theta_{P}\right)\right.$ is $1 / 6 \mathrm{~s}$, and a clinically significant distortion is defined to exceed $0.04 \mathrm{~s}$.

The ventricular activation time (VAT), the time it takes the ventricle to depolarise, is an important clinical parameter. We can estimate this as the time from the onset of the QRS to the intrinsicoid deflection (the sudden downstroke of the QRS). Our surrogate measurement for this is $2 \pi /\left(\theta_{S}-\theta_{R}\right)$. In right orientated leads, a normal VAT is $0.02 \mathrm{~s}$, and on the left (e.g. V6) the duration should not exceed $0.04 \mathrm{~s}$. Therefore, we consider a change of $0.02 \mathrm{~s}$ clinically significant. In the normal ECG used in this paper, the surrogate VAT (QRS width) is $2 \pi /\left(\theta_{S}-\theta_{Q}\right)=0.1 \mathrm{~s}$.

To evaluate the model-fitting performance in the presence of noise a 30s section of ECG is passed through a QRS-detector and a series of beats is segmented around the R-peak $\left( \pm\left|F_{s} \cdot R R_{\max }\right|\right.$ samples, where $R R_{\max }$ is the longest RR interval in the window). Each beat is subsequently time-aligned in a matrix and the residual of a 5component singular value decomposition is computed and reconstructed as a time series. This signal represents the noise in the model, since only the first 5 principal components of an SVD are required to encode the QRS complex [9]. The variance of the signal in the first 5 components over the variance of the residual time series is therefore the SNR. A gradient of a least square line fit of the $\log _{10}$ power and the $\log _{10}$ frequency gives the noise colour $\beta$.

\section{Results}

Experiments titrating noise from from the Physionet Noise Stress Test Data Base [10] show that this is a feasible method of determining noise colouration for electrode motion and baseline wander (black and brown noise). Analysis of the normal sinus rhythm database shows that observation noise is generally pink in nature.

Using these methods, the model-based filter is shown to introduce insignificant clinical distortion in the QT interval and $\mathrm{QRS}$ width down to an $\mathrm{SNR} \geq 0 \mathrm{~dB}$ for $\beta<2$. The fiducial point location is shown to be insignificantly distorted $(<1 \mathrm{~ms})$ for an $\mathrm{SNR} \geq 2 \mathrm{~dB}$, and the ST-level is stable down to SNR $>12 \mathrm{~dB}$. The PR-interval is shown to be more sensitive to noise due to the low amplitude nature of the P-wave. In general, the filter performance is degraded by increasing $\beta$.

\subsection{Applications \& future improvements}

One obvious application of the model-fitting procedure is for ECG-segmentation and feature location. The model parameters explicitly describe the location, height and width of each wave $\left(\theta_{i}, a_{i}\right.$ and $\left.b_{i}\right)$ in the ECG, in terms of a well-known mathematical object, a Gaussian. Therefore, feature locations and parameters derived from these (such as the P-, Q-, and T-onset and hence the PR and QT interval) are easily extracted. Onsets and offsets are conventionally difficult to locate in the ECG, but using a Gaussian descriptor, it is trivial to locate these points as two or three standard deviations of $b_{i}$ from the $\theta_{i}$ in question.

For ECG features that do not explicitly involve the $P$, $Q, R, S$ or $T$ points (such as the ST-segment), the filtering aspect, or noise-free representation of this method is appropriate. If we define the ST segment to be the point of inflection between the $Q$ and $T$ waves, then this point can easily be found as the minimum of the second differential of the segment between the $\theta_{Q}$ and $\theta_{T}$. Furthermore, the $\varepsilon_{r}$ in the fitting procedure provides a confidence index for the extraction of any parameters from the ECG itself.

Another parallel application domain for the model-fit approach is (lossy) compression with a rate of $\left(\frac{F_{s}}{3 k}: 1\right)$ per beat where $k=n+2 m$ is the number of features or turning points used to fit the heart beat morphology (with $n$ symmetric and $m$ asymmetric turning points). For a low $F_{s}$ $(\approx 128 \mathrm{~Hz})$, this translates into a compression ratio greater than 7:1 at a heart rate of $60 \mathrm{bpm}$. However, for high sampling rates $\left(F_{s}=1024\right)$ this can lead to compression rates of almost 60:1.

Although classification of each beat in terms of the values of $a_{i}, b_{i}$, and $\theta_{i}$ is another obvious application for this model, it is still unclear if the clustering of the parameters is sufficiently tight, given the sympathovagal and heart-rate induced changes typically observed in an ECG. It may be 
necessary to normalize for heart-rate dependent morphology changes at least. This could be achieved through utilizing the HR-related compression factor $\alpha$, that we introduced in [4]. However, clustering for beat typing is dependent on population morphology averages for a specific lead configuration. Not only would different configurations lead to different clusters in the 18-dimensional parameter space, but small differences in the exact lead placement relative to the heart would cause an offset in the cluster. A method for determining just how far from the standard position the recording is, and a transformation to project back onto the correct position would be required. One possibility could be to use a procedure designed Moody et al. [9] for their ECG classifier Aristotle. In their approach, the beat clusters are defined in a Karhunen-Loève (KL)-space and therefore an estimate of the difference between the classified KL-space and the observed KL-space is made. Classification is then made after transforming from the observation to classification space in which the training was performed. By measuring the distance between the fitted parameters and pre-trained clusters in the 18-dimensional parameter space, classification is possible. It should be noted that, as with all classifiers, if an artifact closely resembles a known beat, a good fit to the known beat will obviously arise. Therefore setting tolerances on the acceptable error magnitude may be crucial, and a test over a set of labeled databases is required.

\section{Conclusions}

By fitting equation 1 to small segments of the ECG around each QRS-detection fiducial point an idealistic (zero-noise) representation of each beat's morphology is derived. This leads to a method for filtering and segmenting the ECG and therefore accurately extracting clinical parameters even with a relatively high degree of noise in the signal. It should be noted that since the model is a compact representation of oscillatory signals with few turning points compared to the sampling frequency, and it therefore has a band pass filtering effect leading to a lossy transformation of the data into a set of integrable Gaussians distributed over time. This approach therefore could be used on any band-limited waveform. Moreover, the error in each fit can provide beat-by-beat confidence levels for any parameters extracted from the ECG and each fit can run in real time $(0.1$ s per beat on a $3 \mathrm{GHz} \mathrm{P} 4$ processor).

The real test of the filtering properties is not the residual error, but how distorted the clinical parameters of the ECG are in each fit. In this paper, an analysis of the sensitivity of clinical parameters to the colour of additive noise and the SNR is given together with an independent method for calculating the noise colour and SNR. An online estimate of the error in each derived fit can therefore be made. By titrating coloured noise into real ECGs, it has been shown that error in clinical parameters derived from the model-fit method presented in this paper are clinically insignificant in the presence of high amounts of coloured noise. However, clinical features that include low-amplitude features such as the P-wave and the ST-level are more sensitive to noise power and colour. Future research will concentrate on methods to constrain the fit for particular applications where performance is substandard.

\section{Acknowledgements}

GDC acknowledges support by the NIH/NBIB grant R01 EB001659. PEM acknowledges support of a Research Fellowship from the Royal Academy of Engineering and the Engineering and Physical Sciences Research Council.

\section{References}

[1] Biomedical Signal Analysis; A case study approach. IEEE Press, John Wiley \& Sons, 2002; 137-176.

[2] Barros A, Mansour A, Ohnishi N. Removing artifacts from ECG signals using independent components analysis. Neurocomputing 1998;(22):173-18.

[3] McSharry PE, Clifford GD, Tarassenko L. A dynamical model for generating synthetic electrocardiogram signals. IEEE Trans Biomed Eng 2003;50(3):289-294.

[4] Clifford GD, McSharry PE. A realistic coupled nonlinear artificial ECG, BP, and respiratory signal generator for assessing noise performance of biomedical signal processing algorithms. Proc of SPIE International Symposium on Fluctuations and Noise 2004;5467(34):290-301.

[5] Moré JJ. The levenberg-marquardt algorithm: Implementation and theory. In Lecture Notes in Mathematics, volume 630. 1978; .

[6] Clifford GD, Tarassenko L, Townsend N. Fusing conventional ECG QRS detection algorithms with an autoassociative neural network for the detection of ectopic beats. In $5^{\text {th }}$ International Conference on Signal Processing. IFIP, Beijing, China: World Computer Congress, August 2000; 1623-1628.

[7] Wagner G. Marriott's Practical Electrocardiography. $9^{\text {th }}$ edition. Baltimore, Williams \& Wilkins, 1994.

[8] Clifford GD, Tarassenko L. Quantifying errors in spectral estimates of HRV due to beat replacement and resampling. IEEE Transactions in Biomedical Engineering April 2005; 52(4):630-638.

[9] Moody GB, Mark RG. QRS morphology representation and noise estimation using the Karhunen-Loève transform. Computers in Cardiology 1989;269-272.

[10] WWW.physionet.org/physiobank/database/nstdb/.

Address for correspondence:

Gari D. Clifford ( http://alum.mit.edu/www/gari/ )

Harvard-MIT Division of Health Sciences \& Technology

45 Carleton St., / Cambridge MA 02139, / USA 